\title{
Consumo de psicotrópicos sin prescripción médica en farmacodependientes en proceso de rehabilitación
}

\author{
Óscar Medina-Pérez ${ }^{1}$, Luz Adriana Rubio² ${ }^{2}$, Sandra Viviana Ramírez-Carrillo, \\ Mileidy Rodríguez-BernaL ${ }^{2}$
}

\section{Resumen}

Objetivo. Caracterizar el consumo de psicotrópicos sin prescripción médica en farmacodependientes atendidos por una fundación dedicada a la rehabilitación, en el departamento del Quindío, Colombia, entre los años 2006 y 2009.

Métodos. Se utilizó como instrumento para recolectar la información el formulario del Sistema de Vigilancia Epidemiológica para Uso Indebido de Sustancias Psicoactivas -VESPA-, aplicados a los usuarios al momento de ingresar a la institución.

Resultados. En total, 175 personas manifestaron haber consumido psicotrópicos sin prescripción médica; de ellas, $92 \%$ dijo haber consumido Rivotril y $15 \%$ consumieron Rohypnol. Actualmente, 62 consume psicotrópicos y, de ellos, 21\% los ingieren, por lo menos, una vez al día. La edad promedio de inicio de consumo de sustancias psicoactivas fue 12,61 años, en su mayoría, tabaco (43\%); la de psicotrópicos fue 16,9 años.

Conclusiones. Estas cifras sugieren, por un lado, la necesidad de más programas de prevención de consumo de sustancias psicoactivas y, por el otro, de que las autoridades competentes adopten más medidas de control sobre la circulación y el expendio de psicotrópicos.

Palabras clave: abuso de sustancias, epidemiología, factores de riesgo, salud pública.

Magíster, Docente Investigador Universidad de San Buenaventura Medellín en convenio con la FUSM Armenia.

2 Profesional en psicología. Universidad de San Buenaventura, Medellín en convenio con la FUSM Armenia. 


\section{Title}

Consumption of psychotropics without medical prescription drug abusers in rehabilitation process

\begin{abstract}
Objective: characterize the consumption of psychotropic drugs without a medical prescription in drug addict treated by a foundation dedicated to the rehabilitation in the department of Quindío, Colombia, between the years 2006 to 2009 .
\end{abstract}

Methods: The instruments for data collection employed were the Epidemiological Surveillance System for Inadequate Use of Psychoactive Substances' (VESPA),applied to users when entering the institution.

Results: 175 people claim to have used psychotropic drugs without a medical prescription; of these, 92\% said they had consumed Rivotril and $15 \%$ consumed Rohypnol. Actually consume $62 \%$ psychotropic drug, of which $21 \%$ ingested at least once a day. The average age of onset was 12.61 years, in the majority with tobacco $(43 \%)$ of psychotropic to 16.9 .

Conclusions: These figures suggest first, the need to make major prevention programs of psychoactive substance use, another important thing the competent authorities should carry out a more efficient ways of control about the traffic and selling of psychotropic substances.

Key words: substance use, epidemiology, risk factors, public health.

\section{Introducción}

El abuso y la dependencia de sustancias psicoactivas constituyen un grave problema social[1] y de salud pública a nivel mundial, el cual impacta de manera negativa en la salud física y psicológica de quienes las consumen, afectando su vida personal, familiar, escolar, laboral y social[2]. Dicho fenómeno va en aumento y los entes encargados de abordarlo no cuentan con los suficientes recursos para contrarrestar las elevadas tasas de consumo[3].

Diversos estudios han demostrado que las sustancias que se consumen mundialmente con más frecuencia son la marihuana $(54,7 \%)$, las anfetaminas $(32,9 \%)$ y las benzodiacepinas $(25,1 \%)[4]$. En América Latina se estima que 45 millones de ciudadanos consumen la marihuana en mayor proporción que otro tipo de sustancias $y$, aunque las consecuencias fisiológicas que desencadenan las drogas ilegales no son tan graves como las originadas por las drogas legales, las repercusiones sociales son mayores con el uso de las ilegales[5].

Los farmacodependientes generalmente empiezan con el consumo de una o varias sustancias psicoactivas, tales como el tabaco y el alcohol, continúan con marihuana, cocaína, bazuco, sacol o psicotrópicos sin prescripción médica, entre otras sustancias psicoactivas, de las cuales se hace, por lo general, uso simultáneo[6].

En Colombia, el abuso y la dependencia de psicotrópicos benzodiace- 
pínicos se han destacado por ser más frecuente en mujeres que en hombres adultos; sin embargo, actualmente en los adolescentes ya no es tan clara esta distinción[7].

Por otro lado, en el estudio nacional de consumo de sustancias psicoactivas, llevado a cabo por el Gobierno Nacional de Colombia en el año 2008, se encontró que en este país el uso indebido de medicamentos tranquilizantes corresponde a menos de $2 \%$ de los encuestados, quienes declararon haber consumido estas sustancias alguna vez en su vida, con una leve superioridad de consumo por parte de las mujeres. En el universo de este estudio se consideró la población urbana de 19'764.799 de personas de 12 a 65 años, donde se encontró que más de 100 mil personas $(0,52 \%)$ han usado recientemente algún medicamento sin prescripción médica. Asimismo, las mayores cifras de consumo se registran en los grupos de 18 a 34 años y la edad de inicio del consumo de tranquilizantes sin prescripción médica se da a los 22 años, con diferencia de tres años entre hombres y mujeres[8].

El presente estudio busca determinar la prevalencia del consumo de psicotrópicos sin prescripción médica en farmacodependientes atendidos por una fundación dedicada a la rehabilitación en el departamento del Quindío,
Colombia. La importancia del mismo radica en que en dicho departamento no se ha emprendido una investigación de este tipo en población farmacodependiente y, por ende, no se tiene información veraz para desarrollar campañas de prevención más eficaces, entre otras actividades.

\section{Metodología}

\section{Tipo de estudio}

La actual investigación es de carácter cuantitativo, descriptivo y retrospectivo.

\section{Población}

Estuvo compuesta por las 663 personas que entre los años 2006 a 2009 diligenciaron el VESPA al ingresar a una institución de rehabilitación de farmacodependientes en el departamento del Quindío. De ellas, 134 fueron mujeres y 529 hombres, para una relación hombre-mujer de 3,9:1. Las edades reportadas al momento de ingresar fue muy diversa y va desde los 9 hasta los 58 años. Dicha variación se observa además en la gran diferencia que hay entre la media y la mediana: 20,6 y 17,7, respectivamente. Además, se observa un coeficiente de variación de $40,5 \%$ y una desviación estándar de 8,3 años. La edad media de ingreso a la institución fue de 20,6 años. 


\section{Muestra}

Estuvo conformada por todos aquellos usuarios que al diligenciar el VESPA manifestaron que en alguna etapa de su vida consumieron psicotrópicos sin prescripción médica. El rango de la edad de ingreso a la institución fluctuó entre 10 y 45 años; la media se ubicó en 18,80 años, la mediana en 18,32 y la moda en 20,04. La desviación estándar fue de 6,15 años. El $75 \%$ de la muestra ingresó a la institución a una edad máxima de 23,06 años, lo que indica una dispersión alta en la edad de ingreso.

\section{Instrumentos}

Se sistematizaron los formularios del Sistema de Vigilancia Epidemiológica para Uso Indebido pe Sustancias Psicoactivas (VESPA), que los farmacodependientes diligenciaban con el psicólogo de la institución al ingresar a la misma. Dicho instrumento mide las siguientes variables sociodemográficas: fecha de nacimiento, sexo, ciudad y barrio de residencia, nivel educativo, estado civil y ocupación. Además, se preguntaba si había ingresado con anterioridad a un centro de rehabilitación y la razón por la cual en el momento actual estaba en la institución (si llegó por voluntad propia, recomendación de amigos, familiares, indicación legal, etc.); también se indagaba por la droga que primero consumió, su vía de admi- nistración y la edad en que se ingirió por primera vez.

De igual manera, se preguntaba por las otras sustancias que se habían consumido, como sustancias lícitas (tabaco y alcohol), psicotrópicos sin prescripción médica (Rivotril, Rohypnol, Valium, etc.), alucinógenos (marihuana, cocaína, heroína, bazuco, etc.), y otros materiales inhalados, como gasolina. Respecto a estas drogas se indagó sobre la edad de inicio y finalización (en caso de haber dejado de consumirla al momento de hacer la encuesta), la frecuencia de uso (varias veces al día, diariamente, varias veces a la semana, etc.) y la vía de administración (ingerida, fumada, inyectada, inhalada).

\section{Procedimiento}

En una hoja de cálculo de Excel se digitó la información de los formularios VESPA de los usuarios que ingresaron en el periodo 2006-2009. Para garantizar que dicha información fuese fiel a la consignada en físico, la misma fue revisada por un equipo de dos personas que no hubiesen participado en la labor de digitación.

Una vez creada, revisada y ordenada la base de datos del consumo de psicotrópicos, se hizo un análisis descriptivo a las siguientes variables: sexo, nivel educativo, estado civil, ocupación 
$u$ oficio, droga y edad de inicio en el consumo de sustancias psicoactivas, razón por la cual acudió a la institución, existencia o no de tratamiento previo. También, se analizaron la edad de inicio en el consumo de psicotrópicos y la prevalencia actual del consumo de los mismos.

\section{Resultados}

\section{Características sociodemográficas}

Afirmaron haber consumido psicotrópicos sin prescripción médica 175 personas, de las cuales $23,4 \%$ corresponden a mujeres y $76,6 \%$ a hombres.

En la variable escolaridad, se encontró que $1 \%$ no culminó ningún grado escolar, $15 \%$ finalizó los estudios primarios pero no continuó estudiando, mientras que $11 \%$ no terminó la primaria. En cuanto a los estudios secundarios, 49\% no los finalizó y $11 \%$ sí lo hizo. Los estudios técnicos habían sido culminados por $5 \%$, mientras que otro $5 \%$ no los había hecho. El 5\% tenía estudios universitarios incompletos y $1 \%$ no respondió esta información.

Al analizar el estado civil, se halló que $92 \%$ de los consumidores de psicotrópicos refirieron ser solteros, $4 \%$ vivían en unión libre, 3,4\% estaban separados y $0,6 \%$ eran viudos.

En cuanto a la ocupación, se encontró que $66 \%$ no tenían ninguna ocupación, $14,8 \%$ eran estudiantes, $8,5 \%$ tenían trabajo ocasional, $3,3 \%$ tenían trabajo fijo y $7,4 \%$ manifestaron estar desempleados.

\section{Consumo de psicotrópicos sin prescripción médica}

$\mathrm{Al}$ indagar por el consumo de psicotrópicos sin prescripción médica, se encontró que usaban una gran variedad de drogas, como Rivotril, Ativán, Cuait-D, Ritalina, Leponex, Rohypnol, Sinogán y Valium. Entre los medicamentos más consumidos por los farmacodependientes se encontró que el Rivotril fue ingerido por 161 personas, es decir, $92 \%$ del total de la población; de ellos, 35 eran mujeres $(20 \%)$ y 126 hombres (72\%). El segundo medicamento más usado fue el Rohypnol, ingerido por 26 sujetos $(15 \%)$; de ellos, $8(5 \%)$ eran mujeres y $18(10 \%)$ hombres. Se encontró también consumo de Cuait-d $(0,2 \%)$, Valium $(0,1 \%)$ y Ativán $(0,1 \%)$.

$\mathrm{Al}$ indagarse por el consumo actual, se encontró que $38 \%$ ya no la consumía, mientras que el $62 \%$ restante sí lo hacía, con las siguientes prevalencias: mínimo una vez al día, 21\%, mínimo una vez a la semana, $35 \%$, y sin información, $6 \%$. 
En cuanto a la edad de inicio del consumo de psicotrópicos, se encontró que la edad más baja se ubicó en 13 años, mientras que la más alta reportada fue de 25. Se halló también una dispersión alta y, por ello, el coeficiente de variación se ubicó en $86,92 \%$. Ello puede explicar por qué la media, la mediana y la moda son diferentes entre sí: 16,9, 16,2 y 15,21 , respectivamente. Se encontró, igualmente, que $25 \%$ inició el consumo de psicotrópicos a una edad máxima de 13,95 años, $50 \%$ a los 16,2 y $75 \%$ máximo los 19,28 años.

\section{Características del consumo de sustancias psicoactivas - tratamiento}

Se encontró que $61 \%$ refirió no haber recibido tratamiento previo, mientras que el 39\% restante sí lo tuvo.

Al analizar la droga de inicio, se encontró que $43 \%$ de la población empezó consumiendo tabaco, 32\%, marihuana, 14\%, alcohol, y 9\%, cocaína. Ningún sujeto reportó haber empezado con psicotrópicos.

La pregunta que indagaba por las razones que tuvieron los usuarios para asistir a la fundación con el fin de iniciar el tratamiento de rehabilitación, fue respondida por 168 personas (38 mujeres y 130 hombres). De ellos, 86 refirieron haberlo hecho por indicación de amigos o familiares (13 mujeres y 73 hombres); 44 mencionaron que acudieron voluntariamente ( 5 mujeres y 39 hombres) y 35 dijeron que asistieron a la institución por indicación legal (19 mujeres y 16 hombres).

Al revisar la edad de inicio del consumo de sustancias psicoactivas, se encontraron edades muy variadas, las cuales van desde los 5 hasta los 20 años de edad; ello sugiere la existencia de una dispersión alta de la edad, la cual se confirma con el coeficiente de variación hallado: 20,85\%. Por ello, la media, la mediana y la moda son disímiles: 12,61 , 13 y 13,49 , respectivamente. El $25 \%$ empezó a una edad máxima de 11,57 años, el 50\%, máximo a los 13 años y el $75 \%$, a los 15,88 .

Un hallazgo interesante de este estudio es que las mujeres iniciaron el consumo a edades más tempranas que los hombres. Se hace esta afirmación porque, al comparar diversos datos, se encuentran menores edades de inicio en las mujeres. Por ejemplo, al examinar las medias, se halló una diferencia de casi un año: 11,70 frente a 12,66 de los hombres. En la moda ocurre algo similar: 12,35 contra 13,49. También, en los distintos cuartiles sucede lo mismo. En el 1, encontramos 10,07 frente a 10,89 (tabla 1). Se encontró también que a los 15 años el 98\% de las mujeres ya habían iniciado el consumo, mientras que en los hombres a esa edad había empezado a consumir el $87 \%$, lo que reitera una vez más que las mujeres empezaron a edades más tempranas (tabla 1 ). 
Tabla 1

Principales datos estadísticos sobre la edad de inicio, en años

\begin{tabular}{|c|c|c|c|c|c|c|c|}
\hline & Media & Mediana & Moda & Rango & $\begin{array}{l}\text { Cuartil } \\
1\end{array}$ & $\begin{array}{l}\text { Cuartil } \\
2\end{array}$ & $\begin{array}{l}\text { Cuartil } \\
3\end{array}$ \\
\hline Ambos sexos & 12,61 & 13 & 13,49 & 5 a 20 & 11,57 & 13 & 15,88 \\
\hline Hombres & 12,66 & 12,55 & 13,49 & 5 a 20 & 10,89 & 12,55 & 13,78 \\
\hline Mujeres & 11,70 & 12,16 & 12,35 & 6 a 16 & 10,07 & 12,16 & 13,50 \\
\hline
\end{tabular}

Al examinar la edad de ingreso, se encontró que las mujeres asistieron a la institución a edades más tempranas que los hombres, y la comparación de distintos datos estadísticos y cifras así lo dicen: a los 20 años de edad, $92,6 \%$ de las mujeres ya había asistido a la insti- tución, mientras que solo $55,6 \%$ de los hombres lo había hecho. Al examinar los promedios de edad, se ve que el de las mujeres fue de casi 5 años menos: 16 frente a 20,96. Similar condición ocurre en los distintos cuartiles (tabla 2).

Tabla 2

Principales datos estadísticos de la edad de ingreso a la institución, en años

\begin{tabular}{lccccccc}
\hline & Media & Mediana & Moda & Rango & $\begin{array}{c}\text { Cuartil } \\
1\end{array}$ & $\begin{array}{c}\text { Cuartil } \\
2\end{array}$ & $\begin{array}{l}\text { Cuartil } \\
3\end{array}$ \\
& & & & & & & \\
\hline Ambos sexos & 19,80 & 18,32 & 20,04 & 10 a 45 & 15,69 & 18,32 & 23,06 \\
Hombres & 20,96 & 19,24 & 16,34 & 10 a 45 & 16,40 & 19,24 & 22,08 \\
Mujeres & 16,00 & 16,06 & 16,54 & 12 a 24 & 13,86 & 15,57 & 17,03 \\
& & & & & & & \\
\hline
\end{tabular}

Por último, se examinó la edad de inicio del consumo de las principales sustancias reportadas. En este punto se encontró gran variedad de información; por ejemplo, en las sustancias que se reportaron edades de consumo más bajas, fueron el alcohol (5 años), seguido de tabaco y marihuana (6 años), mientras que las más altas fueron para la heroína y el bazuco (10 años). Al revisar las edades promedio de inicio, se encontró que las cifras más bajas fueron para el tabaco y el alcohol (12,28 y 13,52, respectivamente), mientras que la más alta fue para la heroína $(17,59)$ (tabla 3 ). 
Tabla 3

Principales datos estadísticos sobre la edad de inicio de consumo de las distintas sustancias psicoactivas, en años

\begin{tabular}{llllllll}
\hline Droga & Rango & Media & Moda & Sx & $\begin{array}{l}\text { Cuartil } \\
1\end{array}$ & $\begin{array}{l}\text { Cuartil } \\
2\end{array}$ & $\begin{array}{l}\text { Cuartil } \\
3\end{array}$ \\
\hline Tabaco & 6 a 23 & 12,28 & 12,59 & 2,93 & 10,58 & 12,42 & 14,31 \\
Alcohol & 5 a 23 & 13,52 & 14,19 & 3,10 & 11,15 & 13,75 & 15,87 \\
Marihuana & 6 a 20 & 13,69 & 13,87 & 2,69 & 11,44 & 13,75 & 15,88 \\
Cocaína & 8 a 23 & 14,62 & 13,93 & 2,57 & 12,67 & 14,26 & 16,35 \\
Inhalados & 7 a 26 & 14,93 & 12,59 & 3,72 & 12,16 & 14,44 & 17,05 \\
Bazuco & 10 a 27 & 16,51 & 15,42 & 4,10 & 13,45 & 16,04 & 19,28 \\
Heroína & 10 a 37 & 17,59 & 16,7 & 4,88 & 13,53 & 17,23 & 20,63 \\
\hline
\end{tabular}

\section{Discusión}

En el presente estudio se encontró que 175 personas consumieron psicotrópicos sin prescripción médica, entre los que se destacan ansiolíticos como el Rivotril (92\%) y el Rohypnol (15\%). Se halló también, pero en menor proporción, uso de estimulantes, tales como el Cuait-D $(0,2 \%)$, que es un antidepresivo de tipo tricíclico. Este consumo es similar al hallado en un estudio realizado en el eje cafetero por W. Mejía[9], donde se observó que los psicotrópicos de mayor consumo sin prescripción médica fueron Valium, Ativán, Rohypnol y Rivotril. Por otro lado, hay que señalar que el consumo de psicotrópicos para uso no terapéutico puede traer consecuencias nefastas para la salud del ser humano. En el caso de los tranquilizantes o sedantes, pueden generar graves efectos adversos sobre el sistema cardiorrespiratorio[10] y los estimulantes pueden causar temblores, problemas cardiacos, psicosis, hemorragias intracraneales y en muchos casos hasta la muerte[11].

Algunos estudios plantean que el consumo reiterado de estos psicotrópicos podría estar relacionado con el potencial de abuso y adicción que puede generarse en el sistema nervioso central. Su empleo se ha ido incrementando debido a que permiten alcanzar estados de relajación y buen humor, aunque su potencial para provocar euforia no sea tan alto como el de otras sustancias psicoactivas[12].

Además de los efectos químicos de los psicotrópicos, que producen sensaciones de placer, los factores individua- 
les constituyen otro aspecto explicativo acerca del abuso de estas sustancias, es decir, patrones de personalidad que tienden a presentar estados emocionales alterados, tales como trastornos de ansiedad, del estado del ánimo o del sueño, y dificultades para afrontar situaciones valoradas como estresantes y angustiantes o de intenso dolor, en los cuales el uso de psicotrópicos le permite a quien los consume una experiencia de calma y serenidad, o activación según el caso, lo cual es más común en jóvenes que en adultos[13].

También, se debe tener en cuenta la influencia cultural para explicar el origen del uso indiscriminado de psicotrópicos sin prescripción médica, el cual surge con el desarrollo de las sociedades de consumo, como parte de la cultura capitalista que promueve e impulsa a los individuos a hacer uso recreativo de sustancias químicas diseñadas para "equilibrar" estados emocionales o para "intensificar" experiencias de sensación y percepción, que brindan felicidad a las personas, según aseveraciones de los interesados en su comercialización: fabricantes, distribuidores y clínicos[14].

En relación con el sexo, en el presente estudio se encontró que los hombres tienen una prevalencia en el consumo de psicotrópicos de $76,6 \%$, y las mujeres, de $23,4 \%$. Este dato difiere significativamente de otros estudios, como el Estudio nacional de consumo de sustancias psicoactivas (2008), donde se encontró una prevalencia levemente mayor en mujeres $(51,5 \%)$ que en hombres $(48,42 \%)$ [8]. Difiere también de otros estudios realizados recientemente, como el de Oliveira, Paiva y Valente[15] en El Salvador, en el que se demostró que en dicho país el consumo de antidepresivos, tranquilizantes y anfetaminas es mayor en mujeres que en hombres. Algunos estudios aducen que existe una predisposición genética por parte de las mujeres a necesitar tranquilizantes, debido a que su sistema nervioso es más sensible que el de los hombres[16]. Sin embargo, la alta prevalencia de consumo de psicotrópicos en el sexo masculino por encima del femenino en el presente estudio, puede estar relacionado con el historial de consumo. Generalmente, los hombres consumen en promedio más sustancias psicoactivas que las mujeres, debido a sus tendencias fisiológicas hedonistas y deseos de experimentar sensaciones nuevas e intensas con diferentes sustancias, entre ellas los psicotrópicos. Además, los hombres presentan más problemas de socialización, baja tolerancia a la frustración y el dolor, carencia de habilidades para enfrentarse a los problemas cotidianos y exigencias sociales, que las mujeres[17].

En la variable de escolaridad, se encontró que $76 \%$ no había terminado la secundaria, lo cual es una cifra muy preocupante. Asimismo, en un estudio 
anterior se halló una alta deserción entre consumidores[3]; esto puede ser indicador de que el abuso y dependencia a las sustancias psicoactivas afecta el rendimiento escolar, desencadenando abandono de las labores académicas por la necesidad imperiosa de consumir y la falta de motivación para continuar estudiando, debido a que la farmacodependencia es una enfermedad que produce incapacidad[18].

En relación con el estado civil, se encontró que $92 \%$ eran solteros, 4\% vivía en unión libre, $3,4 \%$ estaban separados y $0,6 \%$ eran viudos. Estos porcentajes son diferentes a los hallados en la población general. En el estudio realizado por el Observatorio Departamental de Drogas de Nariño, por ejemplo, se encontró que del total de la población estudiada el 45,3\% declaró ser soltera, el 31,2\% manifestó estar casados, otro 18,1\% convivía en unión libre, 2,6\% respondió estar separado, 1,9\% se encontraba en periodo de viudez y el $0,4 \%$ restante no respondió sobre su estado civil[19]. Ello sugiere que probablemente los consumidores de sustancias psicoactivas poseen dificultades para establecer y mantener relaciones duraderas de pareja, pues se ha observado una relación directa entre maltrato, disfunción familiar y la dependencia a las sustancias psicoactivas, debido a cambios significativos en el comportamiento que tienden a ser violentos y desadaptados[20].
Con respecto al nivel de ocupación, el presente estudio muestra que la mayoría de la población (66\%) no tenía ninguna ocupación, es decir, no realizaban actividades de tipo laboral, académica o lúdica. Tan solo 14,8\% eran estudiantes, cifra que es bastante inferior en relación con el total de la población, y solo el 3,3\% tenían trabajo fijo. En Nariño se encontró una importante diferencia, donde el Observatorio Departamental de Drogas reportó que 49,97\% de la población se encontraba trabajando, 18,65\% laboraba en servicios domésticos, $8,85 \%$ manifestaba no trabajar, $0,35 \%$ estaba jubilada o pensionada, 3,92\% se dedicaba a buscar trabajo, $16,16 \%$ se encontraba estudiando y $0,35 \%$ eran discapacitados; el 0,86\% no respondió a esta variable[19]. En efecto, la desocupación o desvinculación social del individuo, hace parte de los factores de riesgo para el consumo de sustancias psicoactivas, debido a que es común encontrar en los diferentes sectores populares que los jóvenes son excluidos de mecanismos de participación política, producción económica, social y cultural de una nación, lo cual reduce las expectativas personales de futuro y oportunidad, y desencadena conductas delincuenciales, perturbadoras y violentas[21].

Al estudiar la edad de ingreso a la institución, se encontró que las mujeres buscaban ayuda a edades más tempranas que los hombres: 16,00 años frente 
a 20,96 años de los hombres. Estas diferencias pueden responder a los estereotipos de masculinidad que promueven la inexpresividad emocional y dificultan la búsqueda de ayuda[22].

En síntesis, este estudio es un punto de partida para las investigaciones en este campo en el departamento, las cuales son bases fundamentales para la creación, desarrollo y fortalecimiento de programas de prevención. Particularmente, llama la atención la edad temprana de inicio, lo cual se considera como un factor de riesgo, puesto que estudios anteriores han demostrado que, a menor edad, mayores son las consecuencias en el desarrollo físico, mental y social[23].

Durante la etapa de la preadolescencia y la adolescencia, se presenta mayor riesgo para el inicio del consumo de sustancias[7], debido a que son dos etapas donde el individuo experimenta cambios físicos, emocionales y sociales, en donde el factor psicológico es el de mayor impacto; en este se desarrolla su nueva identidad, nuevas habilidades para ver las cosas en términos relativos y abstractos, es decir, empiezan a desarrollar su repertorio básico de conducta para tomar decisiones, que puede en algunos casos ser riesgoso y atentar contra su seguridad.

Con frecuencia los adolescentes se enfrentan a nuevos desafíos y retos, lo que generalmente los lleva a adoptar conductas de abuso y dependencia a sustancias psicoactivas[24]. Además, es importante considerar la influencia del grupo de pares en este ciclo vital, en el que la experimentación con sustancias psicoactivas representa una motivación por saciar deseos hedonistas, modelos negativos, redefiniciones de la identidad personal y psicosocial[25]. Es así como se hace necesario que las instituciones públicas y privadas, entre otros organismos, se interesen, además de la rehabilitación y resocialización, en la prevención del consumo de sustancias psicoactivas en edades tempranas, puesto que es una etapa crítica del ser humano, en que se le debe prestar mayor atención, implementando estrategias educativas orientadas al establecimiento de valores, normas y principios que generen en el individuo crecimiento personal y le permitan proyectarse positivamente hacia el futuro[26].

\section{Conclusiones y recomendaciones}

Los resultados del presente estudio pueden ser significativos para el desarrollo de programas más efectivos de promoción y prevención de consumo sustancias psicoactivas, especialmente el de psicotrópicos, ya que describen altas cifras halladas en el uso de dichas sustancias y se pone de relieve la necesidad de realizar estudios más rigurosos, que permitan descubrir los factores que 
están directamente relacionados con el incremento de este consumo, permitiendo así desarrollar propuestas eficaces para su prevención y reducción.

Mediante este estudio, se busca ilustrar no solo a las autoridades y organismos gubernamentales para que amplíen las medidas de control sobre la circulación y expendio de psicotrópicos, ya que en la actualidad es evidente que se está fallando en este aspecto, sino que además, es necesario hacer reflexionar a la sociedad en general acerca de la gravedad del alto consumo de psicotrópicos sin prescripción médica, sin dejar de lado la prevalencia del consumo de sustancias legales como el tabaco y el alcohol, identificadas como sustancias inductoras, pues son las más usadas por primera vez, y las ilegales, como el bazuco, marihuana, cocaína y heroína, las cuales causan daños irreversibles en la salud.

Además, es importante crear conciencia sobre los efectos adversos del uso de medicamentos psicotrópicos en el organismo, evitando así que sus fabricantes, comercializadores y personal médico continúen promoviendo la masificación de su consumo, al sugerir que la medicación es la manera más sencilla de sentirse mejor.

\section{Bibliografía}

1. Jáuregui I. Droga y sociedad. La personalidad adictiva de nuestro tiempo. Nómadas:
Revista crítica de ciencias sociales y jurídicas. 2007, 7. Disponible en: http://www. ucm.es/info/nomadas/16/ijbalenciaga.pdf.

2. Campollo O. Una estrategia transversal para resolver un problema de salud pública: las adicciones. El Observador. 2005, 3(3). Disponible en: http://www.cicad.oas. org/oid/new/information/elobservador/ ElObservador3_2005/NHSNDA.pdf.

3. Correa M. Relación e impacto del consumo de sustancias psicoactivas sobre la salud en Colombia. Bogotá: Universidad Católica de Colombia. Tesis de grado. 2009 Disponible en: http://odc.dne.gov. co/docs/publicaciones_nacionales/TESIS_DE_GRADO_ucatolica.pdf

4. Urrego D. Consumo de sustancias psicoactivas en estudiantes de especialidades médicas, Bogotá 2001. Rev Salud Pública. 2002;4: 59-73. Disponible en: http:// www.revmed.unal.edu.co/revistasp/v4n1/ v4n1a2.htm

5. Peruga A, Rincón A, Selin H. Guías para el diagnóstico y tratamiento de las principales drogas de abuso. Revista Electrónica de las Ciencias Médicas en Cienfuegos. 2003;1:47-62. Disponible en: http://www. medisur.sld.cu/index.php/medisur/article/ view/34/4464

6. Vargas D. Alcoholismo, tabaquismo y sustancias psicoactivas. Rev Salud Pública. 2001;3. http://www.revmed.unal.edu.co/ revistasp/v3n1/Rev316.htm

7. Martínez J, Amaya W, Campillo H, Rueda G, Campo A, Díaz L. Consumo de sustancias psicoactivas en adolescentes, Bucaramanga, Colombia, 1996-2004. Rev Salud Pública. 2007;9:215-29. Disponible en: http://www. scielosp.org/pdf/rsap/v9n2/v9n2a06.pdf

8. Ministerio de la Protección Social. Estudio nacional de consumo de sustancias 
psicoactivas. Bogotá: Guadalupe. 2009 Disponible en: http://www.corazonesresponsables.org/Estudio_Nal_Completo_ Psicoactivas.pdf

9. Mejía W. Drogas psicoactivas reportadas como consumidas en el eje cafetero y léxico asociado a ellas, en un estudio de percepciones. Revista Cult Drog Red. 2006;13:275-323. Disponible en: http://200.21.104.25/culturaydroga/downloads/culturaydroga $11 \% 2813 \% 29$ _13.pdf

10. Muriel C, García M, Yusta G, Sánchez F. Citrato de fentanilo transmucosa oral. Rev Soc Esp Dolor. 2000;7:319-26. Disponible en: http://revista.sedolor.es/pdf/2000_05_ 08.pdf

11. Montes R, Correa T, Granda M. Las efedrinas como estimulantes del sistema nervioso central y su implicación en el deporte. Disponible en: http://www.imd. inder.cu/adjuntos/article/132/Las\%20efed rinas $\% 20$ como $\% 20$ estimulantes.pdf

12. Borrás R, Boada S, Casamitjana N, Casas X, Guayta R, Gironella C, et al. Abuso de medicamentos: ¿la adicción del siglo XXI? FMC. 2007;14:255-62. Barcelona, España. Disponible en: http://www.elsevier.es/sites/default/files/elsevier/pdf/45/ 45v14n05a13102542pdf001.pdf

13. Pinos N, Inocenti A, TirapelliII C. Consumo de benzodiacepinas sin prescripción médica en los/as estudiantes de primer año de la escuela de enfermería de la Universidad de Guayaquil, Ecuador. Escuela de Enfermería de Ribeirão Preto de la Universidad de São Paulo, Centro Colaborador de la OMS para el Desarrollo de la Investigación en Enfermería, Brasil Rev. Latino-Am. Enfermagem v. 16 no.spe Ribeirão Preto July/Aug. 2008. Disponible en: http://www.scielo.br/scielo. php?pid=S0104-1692008000700021\&scr $\mathrm{ipt}=$ sci_arttext\&tlng=es
14. Mazzotti P. Las drogas, sus implicaciones culturales, políticas y económicas Universidad Jaume. Jornades de Foment de la Investigación. Disponible en: http://www. uji.es/bin/publ/edicions/jfi5/drogas.pdf

15. Oliveira J, Paiva M, Valente C. La intervención del contexto asistencial en la visibilidad del consumo de drogas por mujeres. Revista Latino-am Enfermagem. 2007;15(2). Disponible en: http://www. scielo.br/scielo.php?pid=S0104-1169200 $7000200009 \&$ script $=$ sci_arttext\&tlng=es

16. Pistani M, Ruiz V. El consumo indebido de medicamentos psicotrópicos en la vida cotidiana. Un estudio exploratorio sobre representaciones sociales y patrones de uso. Observatorio Argentino de Drogas. Sedronar. 2007. Disponible en: http:// www.observatorio.gov.ar/investigaciones/ MedicVidaCot.pdf

17. Rivas R, Jenner C. Sustancias psicoactivas lícitas (alcohol y tabaco) en los estudiantes masculinos y femeninos de 16 a 45 años de edad que cursan entre el primero y quinto año de estudio durante el ciclo 01 del año 2004 en la Universidad Francisco Gavidia de San Salvador, 2004. Disponible en: http://www.javeriana.edu. co/redcups/Tesis_diagnostico_prevalencia_universitarios.pdf

18. Cote M, Leal E, Prieto E, Vargas D. Relación entre el consumo crónico de sustancias psicoactivas y alteraciones neurocomportamentales en fármaco-dependientes en rehabilitación en comunidades terapéuticas (feccot). Bogotá Cundinamarca 20062007. Estudio descriptivo. Disponible en: Rev Fac Med. 2008;56:338-52. http:// www.revistas.unal.edu.co/index.php/revfacmed/article/viewFile/14491/16901

19. Observatorio Departamental de Drogas. Consumo de drogas lícitas e ilícitas en el departamento de Nariño 2006-2007. 
Investigación Cuantitativa. Pasto, 2008. Disponible en: http://www.descentralizadrogas.gov.co/investigaciones/Qconsumo_\%5B1\%5D...pdf

20. Cáceres D, Salazar I, Varela M, Tovar J. Consumo de drogas en jóvenes universitarios y su relación de riesgo y protección con los factores psicosociales. Univ. Psychol. v. 5 n. 3 Bogotá, dez. 2006. Disponible en: http://pepsic.bvsalud.org/scielo. php?pid=S1657-92672006000300008\&sc ript=sci_arttext\&tlng=en

21. Cáceres N. Experiencia perceptiva de sí mismo, de su familia y comunidad en un grupo de jóvenes del barrio el Vergel de la Comuna 13 de Santiago de Cali. Rev Pensamiento Psicológico. 2006;2:149-68. Disponible en: http://revistas.javerianacali.edu.co/index.php/pensamientopsicologico/article/view/274/308

22. Sánchez R, Tejada P, Guzmán Y. Muertes violentas intencionalmente producidas en Bogotá, 1997-2005: diferencias según el sexo. Rev Colomb Psiquiatr. 2008;37:316-29. Disponible en: http:// www.psiquiatria.org.co/BancoMedios/ Documentos\%20PDF/rcp3artorig1_muertes_violentas_intencionalmente.pdf

23. Macleod J, Oakes R, Copello A, Crome L, Hckman M, Oppenkowski T. et al. Consecuencias psicológicas y sociales del Can- nabis y otras drogas ilícitas consumidas por los jóvenes: informe sistemático de estudios longitudinales de la población general. Rev Toxicomanías. 2005;43:11-22. Disponible en: http://www.cat-barcelona. com/pdfret/RET43-2.pdf

24. Burrone M, Villela S, Costa de M, Enders J. Fernández R, Pereira G. Análisis de la frecuencia de experimentación y consumo de drogas en alumnos de escuelas de nivel medio. Rev Latino-Am. Enfermagem. 2010;18(spe):648-54. Disponible en: http://www.scielo.br/scielo. php?script=sci_arttext\&pid=S0104-1169 2010000700023\&lang=pt

25. Moral M, Ovejero A. Modificación de las actitudes, hábitos y frecuencia de consumo de alcohol y otras sustancias psicoactivas en adolescentes españoles a partir de un programa educativo-preventivo. Rev Colomb Psicol. 2005;14:100-18. Disponible en: http://www.revistas.unal.edu. co/index.php/psicologia/article/viewFile/1224/1775

26. Medina N, Carvalho M. Factores protectores de las familias para prevenir el consumo de drogas en un municipio de Colombia. Rev. Latino-Am. Enfermagem. 2010;18(spe):504-12. Disponible en: http://www.scielo.br/scielo. php?script=sci_arttext\&pid=S0104-1169 2010000700004\&lang=pt. 